\title{
ESTIMATION OF RF PA NONLINEARITIES AFTER CROSS-CORRELATING POWER SUPPLY CURRENT AND OUTPUT VOLTAGE
}

\author{
Ricardo Veiga, Pedro Mota, José Machado da Silva \\ INESC Porto, Faculdade de Engenharia, Universidade do Porto \\ Rua Dr. Roberto Frias, Campus FEUP, 4200-465 Porto, Portugal \\ [ee08064, dee05001,jms]@ fe.up.pt
}

\begin{abstract}
The present paper describes developments carried-out on estimating $1 \mathrm{~dB}$ compression and thirdorder intercept points after the cross-correlation between power supply dynamic current and output voltage of radiofrequency power amplifier. The underlining theory and a circuit that allows implementing this measurement on-chip are presented. Simulation results, including the analysis of optimum stimuli amplitudes and the Monte Carlo analysis to circuits' process variations are presented. These show that good accuracy can be obtained with relatively simple measurement conditions.
\end{abstract}

\section{INTRODUCTION}

The search for on-chip RF testing methods has motivated different research activities in the past decade [1-6]. Nonlinearity estimation is among the most important parameters which have driven this research [7,8]. That is the case with circuits operating with digital modulations such as Wide-bandwidth Code-Division for Multiple Access (W-CDMA), which presents a high degree of amplitude variation or, in other words, a high crest factor $(\mathrm{CF})$. High linearity levels are required for transceivers operating in these conditions to prevent signal compression or clipping.

Traditional RF testing relying on high-end laboratory instruments provide accurate measures but are time consuming and expensive. On the other hand, accessing deeply embedded circuits requires delicate electronic probes. This is particularly true within modern systems-onchip (SoC) devices that incorporate different circuits and functionalities on a monolithic substrate, such as, highspeed digital, memory, analog, and RF circuits. This high integration of components makes SoC testing complex and costly [9].

The lack of harmonized and universal RF structural tests have forced industries to resort to specifications' functional testing in order to ensure the required quality level of low defect ppm (ideally zero), eventually relying on both builtin and built-off test $[9,10]$. The methodologies presented in [12,13] allow testing a RF power amplifier (PA) for nonlinearity using a polynomial fitting approach, after applying single tone input stimuli sweeping the PA's dynamic range and on capturing the respective output values. From this set of coordinates one can obtain the polynomial that best fits the PA's input/output characteristic and whose coefficients allow one to calculate $1 \mathrm{~dB}$ compression ( $\left.\operatorname{Pin}_{1 d B}\right)$ and third order intercept ( Pin $_{I I P 3}$ ) points values.

The methodology being presented here relies also on the polynomial fitting approach, however, ordinates are provided by the cross-correlation between output voltage and power supply current, instead. The remaining of the paper presents in section II the procedure being proposed to estimate $\operatorname{Pin}_{1 d B}$ and $\operatorname{Pin}_{I I P 3}$ from the power transfer characteristic curve, together with validating simulation results. Section III shows how power measures can be obtained from the cross-correlation between voltage and current signals, as well as, the mixer based circuit being used as a correlator. Section IV highlights the main conclusions.

\section{ESTIMATION OF NONLINEARITY}

It has been shown that a weakly nonlinear, memoryless, time-invariant, PA's behaviour can be expressed by a thirdorder polynomial [11]. This fact has been explored to develop in-circuit test circuits based in the observation of the input and output RF voltage signals [4, 12, 13]. Likewise, one can estimate the PA's power transfer characteristic after finding the polynomial $P_{\text {out }}=\beta_{1}$ Pin $+\beta_{2}$ Pin $^{2}+\beta_{3}$ Pin $^{3}$ that best fits a set of observed input-output power pairs, and whose coefficients allow us to compute $\operatorname{Pin}_{1 d B}$ and $\operatorname{Pin}_{I I P 3}$ points. Following procedures similar to those presented in [11-13] one can obtain, respectively,

$$
\operatorname{Pin}_{1 d B}=\frac{-\beta_{2}-\sqrt{\beta_{2}^{2}-4 \beta_{3}\left(\beta_{1}-\frac{\beta_{1}}{10^{\frac{1}{10}}}\right)}}{2 \beta_{3}} .
$$

and $\operatorname{Pin}_{I I P 3}=\sqrt{\left|\frac{\beta 1}{\beta 3}\right|}$ to estimate $\operatorname{Pin}_{1 d B}$ and $\operatorname{Pin}_{I I P 3}$. 
To obtain the coefficients of the best fitting polynomial the following procedure was followed. If one obtains three output power levels from the PA under test $\left(P_{\text {out } 1,2,3}\right.$ in figure 1 ), the values of the three coefficients $\beta_{1}, \beta_{2}$ and $\beta_{3}$, can be obtained from the set of equations (2.a and 2.b)

$$
\left\{\begin{array}{l}
\text { Pout } 1=\beta 1 \text { Pin } 1+\beta 2 P \text { Pin } 1^{2}+\beta 3 P \text { Pin } 1^{3} \\
\text { Pout } 2=\beta 1 \text { Pin } 2+\beta 2 P \text { Pin } 2^{2}+\beta 3 \text { Pin } 2^{3} \\
\text { Pout } 3=\beta 1 \text { Pin } 3+\beta 2 P \text { Pin } 3^{2}+\beta 3 \text { Pin } 3^{3}
\end{array}\right.
$$

which after applying Cramer's rule allows us to obtain

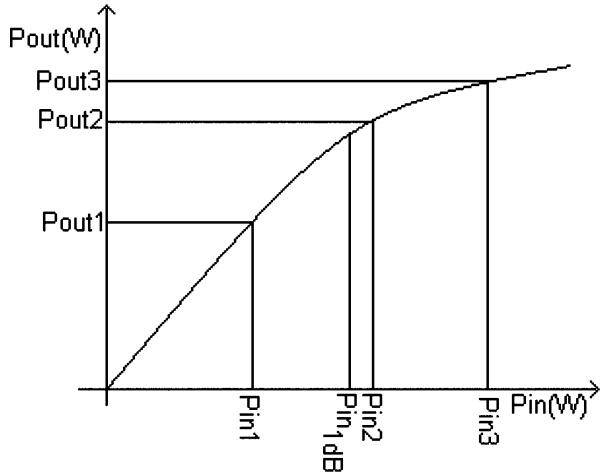

Figure 1 - Weakly nonlinear, memoryless PA's transfer characteristic.

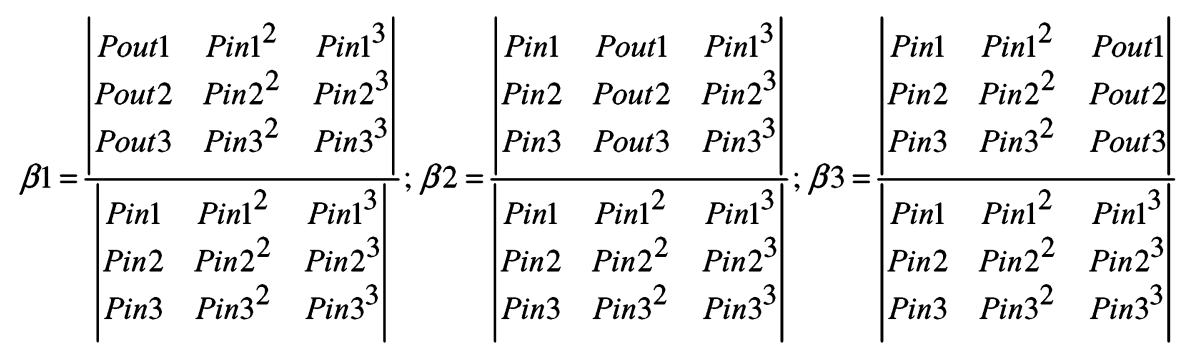

Nevertheless, the three testing stimuli $P_{\text {in } 1,2,3}$ have to be optimized in order to obtain the best estimation accuracy for $P_{i n} 1 d B$ and Pin $_{I I P 3}$. To find the best set of three stimuli to be used a minimum search function $\min _{x} f(x)$, where $x$ is the vector of the three stimuli amplitudes, and $f(x)$ is the error in the computation of $\operatorname{Pin}_{1 d B}$, was employed.

Simulation results obtained within the Agilent ADS tool using a class $\mathrm{AB}$ PA, revealed that choosing $\operatorname{Pin}_{1}$ and Pin $_{3}$ in order that $P i n 1<\operatorname{Pin}_{1 d B}<$ Pin3, and $\operatorname{Pin}_{2}$ close to the expected $P_{i n_{1}}$ (figure 1), a very accurate $P i_{1} d B$ estimation can be obtained. Figure 2 allows one to compare the real power transfer curve against the extrapolated found with this procedure, and reveals that a very good match is obtained.

Figure 3.a) shows the variation of the $\operatorname{Pin}_{1 d B}$ estimation error with the placement of the three stimuli in relation to the expected Pin $1 d B$, keeping the distance among them constant. It can be seen that when the middle value of the three stimuli is close to the expected $\operatorname{Pin}_{1 d B}$, the error is very small. In fact, practically equal estimated and expected values were obtained, i. e.,
Locating the intermediate stimulus in this zone allows also to minimize the deviation due to random noise in voltage and current signals, as can be seen in figure 3.b), which was obtained after superimposing random noise, with a $30 \mathrm{~dB}$ signal to noise ratio, in the correlator's input signals. Similar conclusions were obtained with different power transfer curves.

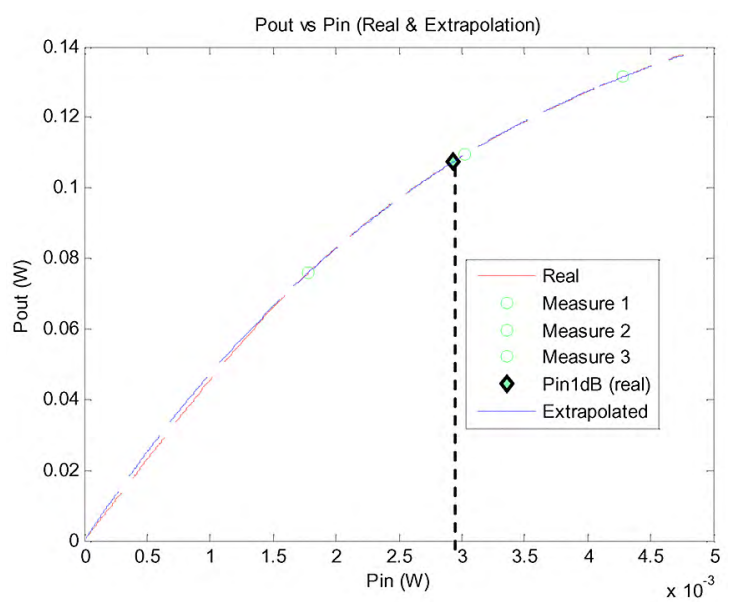

Figure 2 - Power transfer curves obtained with a class AB amplifier.

$$
\operatorname{Pin}_{1 \mathrm{dBest}} \approx \operatorname{Pin}_{1 \mathrm{~dB} \exp }=4,67 \mathrm{dBm}
$$




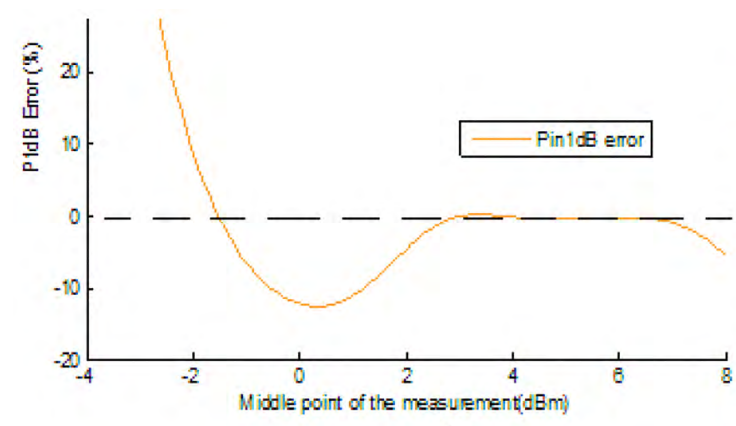

a)

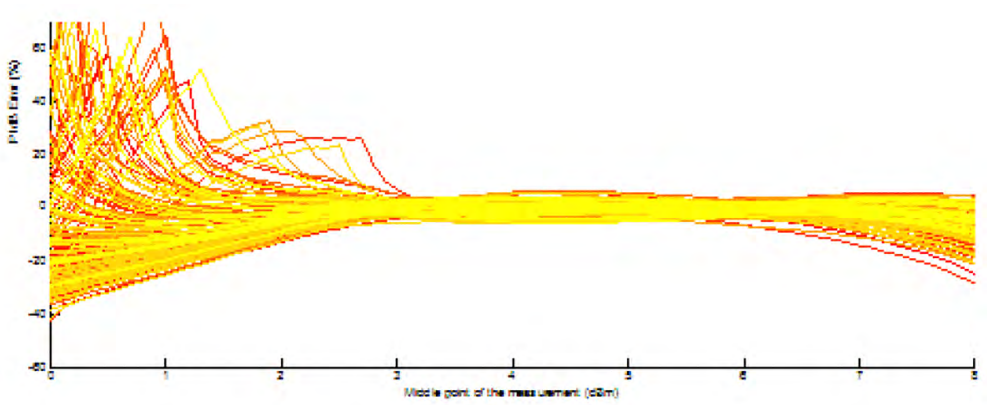

b)

Figure 3 - $\operatorname{Pin}_{1 d B}$ estimation error.

Concerning the estimation of IIP3 small errors can also be obtained but the sensitivity to the placement of the three stimuli amplitude in the transfer characteristic is higher (figure 4) - anyway the obtained estimated value is Pin $_{\text {IIP3est }}=12.5 \mathrm{dBm}$ while the expected one is $P_{\text {in }}$ IIP 3 exp $=13 \mathrm{dBm}$, i. e., the error of $3.8 \%$ occurs.

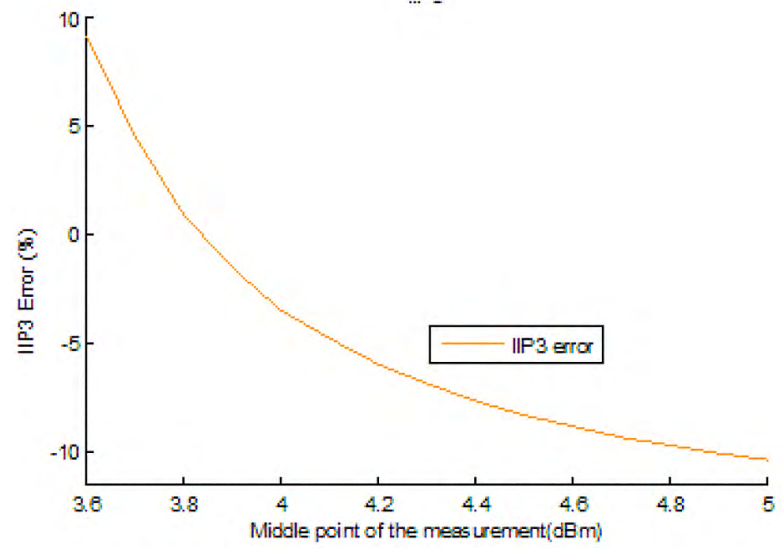

Figure $4-$ Pin $_{I I P 3}$ estimation error.

It is shown in $[12,13]$ that IIP3 estimation from PA's gain compression curves provides results with acceptable accuracy, but which may eventually require considering higher order fitting polynomials. Further work will be carried-out on this matter.

\section{III - CROSS-CORRELATION BASED POWER MEASUREMENT}

Looking at voltage amplitudes, as it has been done in other works, does not provide a fully accurate power measure in case an impedance deviation has occurred. This requires observing the actual power in the nodes being observed. The cross-correlation between two signals, $x(t)$ and $y(t)$ is defined as $R(\tau)=\int^{+\infty} x(t) * y(t+\tau) d t$, where $\tau$ is a time delay, which becomes

$$
R(\tau)=\frac{1}{2} X Y \cos (\omega \tau+\theta)
$$

in the case $x(t)$ and $y(t)$ are periodic signals of period $\mathrm{T}$, given by $x(t)=X \sin (\omega t)$ and $y(t)=Y \sin (\omega t+\theta)$. If $x(t)$ and $y(t)$ are the voltage and current of a circuit, (3) gives us the circuit's active power when time delay $\tau$ is null. That is,

$$
\begin{aligned}
& \Re v i(0)=\frac{1}{2} V I \cos (\theta) \equiv \\
& P=\frac{1}{T} \int_{0}^{T} V(t) \times I(t) d t=\frac{1}{2}(V \times I) \times \cos (\theta)
\end{aligned}
$$

The correlation circuit implemented with a mixer and a low-pass filter shown in figure 5 was used to evaluate the applicability of the cross-correlation between power supply current and output voltage to estimate $\operatorname{Pin}_{1 d B}$ and Pin $_{I I P 3}$. The voltage and current signals are filtered in order to observing only the respective fundamental components, $v_{o u t_{1}}$ and $i_{d d_{1}}$.

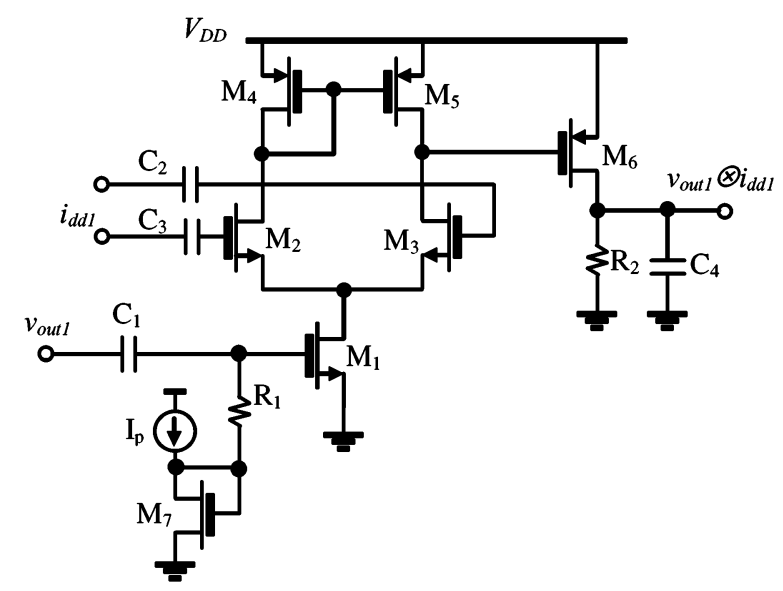

Figure 5 - Correlator circuit.

Figure 6 shows the correlator's output voltage as a function of the power to be measured. It can be seen that a 
linear correspondence exists between the correlator output voltage and the power to be measured.

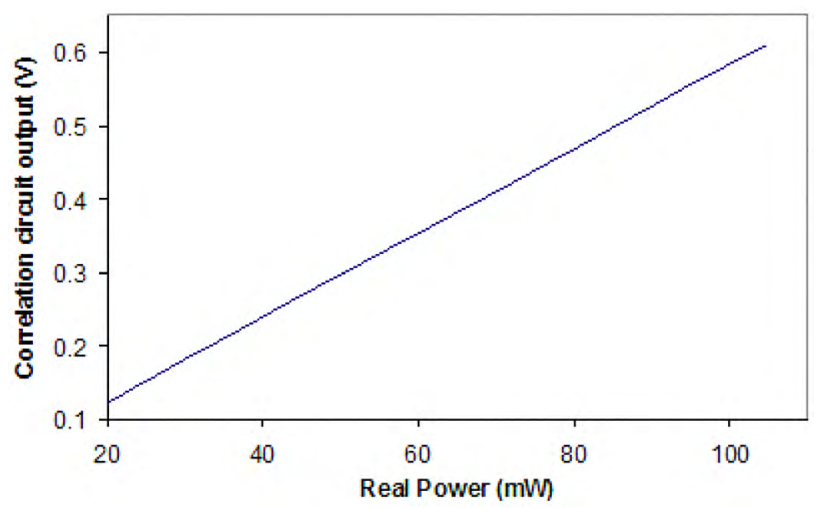

Figure 6 - Correlator output voltage vs. PA's output power.

This is also seen in figure 7, which shows both the PA's actual transfer characteristic $\left(P_{\text {out }}\right.$ real) and that obtained with the correlation based sensor. The good matching between the two curves allows us to estimate $\operatorname{Pin}_{1 d B}$ and $P_{\text {in }}$ IIP3 with the same accuracy, i.e.,

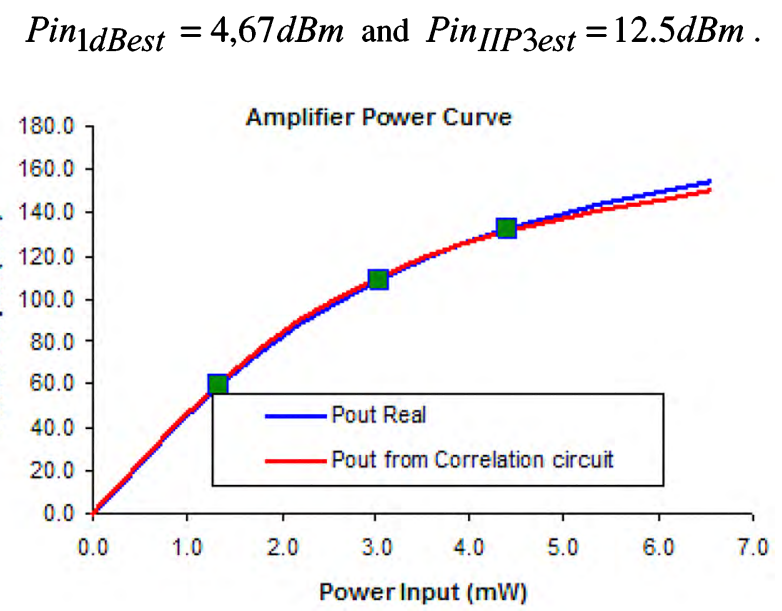

Figure 7 - Comparison between the actual power transfer curve and that obtained with the correlator sensor.

\section{IV - CONCLUSIONS}

The development of built-in self-test solutions for radiofrequency circuits has not yet reached the required maturity to help replacing comprehensive but time-consuming characterization testing, for tests capable to screen malfunctioning circuits due to processing defects and process variations. The work presented herein addresses the estimation of RF amplifiers' $1 \mathrm{~dB}$ compression and thirdorder intercept nonlinearity figures after cross-correlating dynamic output voltage and power supply current. This allows estimating nonlinearity parameters using actual amplifiers' output power measures, instead voltage measures has it has been proposed before. A simple mixer and low-pass filter circuit is used for this purpose. Simulation results are presented which show that good accuracy estimations can be obtained with just three stimuli levels.

\section{REFERENCES}

[1] M. Hafed, N. Abaskharoun, and G. Roberts, "A StandAlone Integrated Test Core for Time and Frequency Domain Measurements", Proceedings of the International Test Conference, 2000.

[2] John Ferrario, Randy Wolf, Steve Moss, and Mustapha Slamani, "A Low-Cost Test Solution for Wireless Phone RFICs", IEEE Communications Magazine, September 2003.

[3] Jee-Youl Ryu, Bruce C. Kim, Senior Member, IEEE, and Iboun Sylla, "A New Low-Cost RF Built-In Self-Test Measurement for System-on-Chip Transceivers", IEEE Transactions on Instrumentation and Measurement, Vol. 55, No. 2, Page(s):381 - 388April 2006.

[4] Valdes-Garcia, A.; Silva-Martinez, J.; Sanchez-Sinêncio, E.; "On-Chip Testing Techniques for RF Wireless Transceivers", IEEE Design \& Test of Computers, Vol. 23, No. 4, pp. 268 - 277, April 2006.

[5] Fan, X.; Onabajo, M.; Fernandez-Rodriguez, F. O.; SilvaMartinez, J.; Sanchez-Sinêncio, E.; "A Current Injection Built-In Test Technique for RF Low-Noise Amplifiers", IEEE Transactions on Circuits and Systems I, Vol. 55, No. 7, pp. 1794 - 1804, Aug. 2008.

[6] Voorakaranam, R.; Akbay, S. S.; Bhattacharya, S.; Cherubal, S.; Chatterjee, A., "Signature Testing of Analog and RF Circuits: Algorithms and Methodology", IEEE Transactions on Circuits and Systems I, Vol. 54, No. 5, pp. 1018 - 1031, May 2007

[7] R. Hassun, N. Kuhm, R. Posner, R. Sweeney, B. Vassilakis, "Ultra-linear Power Amplifier Characterization Using Dynamic Range Extension Techniques", IEEE Symposium MTT, 2001.

[8] Guillot, Farah; Garcia, Patrice; Mouis, Mireille; Belot, Didier; "Analysis of the intermodulation distortion and nonlinearity of common-base SiGeC HBTs. 13th IEEE International Conference Electronics, Circuits and Systems, ICECS '06. pp. 664 - 667, 10-13 Dec. 2006.

[9] Strid, Eric W.; "High-Throughput RFIC Wafer Testing" ARFTG Conference Digest-Spring, $57^{\text {th }}$ Vol. 39, pp. $1-5$, May 2001.

[10] Ellouz, S.; Gamand, P.; Kelma, C.; Vandewiele, B.; Allard, B.; "Combining Internal Probing with Artificial Neural Networks for Optimal RFIC Testing", IEEE International Test Conference, pp. 1 - 9, Oct. 2006.

[11] B. Razavi, RF Microelectronics, Englewood Cliffs, NJ, Prentice Hall, 1998.

[12] Choongeol Cho, William R. Eisenstadt, Bob Stengel, and Enrique Ferrer, "IIP3 Estimation From the Gain Compression Curve", IEEE Trans. On Microwave Theory and Techniques, Vol. 53, No. 4, April 2005.

[13] Pedro Mota, José Machado da Silva, and John Long, "Estimation and Adaptive Correction of PA's Nonlinearities", 13th International Mixed Signals Testing Workshop and 3rd GHz/Gbps Test Workshop, June 2007. 